Сташевский 3.П. / Stashevskiy Z.P. ${ }^{1}$

д-р техн. наук, проф. Грыцюк Ю.И. / prof. Grytsyuk Yu.I. ${ }^{2}$

Przyjęty/Accepted/Принята: 06.06.2014;

Zrecenzowany/Reviewed/Рецензирована: 11.02.2015;

Opublikowany/Published/Опубликована: 31.03.2015;

\title{
Основные компоненты проекта подготовки специалистов по информационной безопасности в учебных заведениях с особыми условиями обучения ${ }^{3}$
}

\author{
Major Project Components of Specialist Training in Information Security \\ in Educational Institutions with Special Training Conditions
}

\author{
Główne elementy projektu przygotowania specjalistów w dziedzinie bezpieczeństwa \\ informacji na uczelniach ze specjalnymi warunkami kształcenia
}

\begin{abstract}
АННО ТА ИЯ
Цель работы: Проанализировать структуру и определить основные компоненты формирования проекта подготовки специалистов по информационной безопасности (ИБ) образовательно-квалификационного уровня бакалавр в учебных заведениях с особыми условиями обучения, как составного звена обеспечения качественного образовательного процесса. Разработать математическую модель требований к образовательно-квалификационной характеристике (ОКХ) и образовательно-профессиональной программе (ОПП), что даст возможность компонировать сбалансированные циклы дисциплин для формирования у выпускника ВУЗ затребованных компетенций от производителей. Введение: Имеющиеся на сегодня АСУ в структурных подразделениях ГСЧС Украины предназначены для обработки больших объемов информации, для обеспечения ее хранения и передачи между всеми звеньями спасательной службы, результатом чего является своевременная, сбалансированная и бесперебойная работа самой службы. Но, как и любая информационная система (ИС), принимая во внимание проявление злоумышленников в информационной среде, она должна быть защищена от них. Из проведенного нами анализа источников угроз и уязвимостей ИС видно, что человеческий фактор и уровень подготовки персонала имеют ключевое значение при качественном построении и бесперебойном функционировании систем защиты информации в ГСЧС Украины. Для решения указанных проблем необходимо создание соответствующей ведомственной гибкой системы подготовки специалистов в области ИБ на базе ВУЗ ГСЧС Украины с особыми условиями обучения. Эта система базируется на принципах современного высшего образования Украины, реализованных в положении “Болонская декларация в системе высшего профессионально-технического образования Украины” на основе компетентностного подхода, предусматривающего тесное взаимодействие когнитивного рынка трудоустройства и ведомственных учебных заведений. Выводы: Проведен анализ структуры и определены основные компоненты построения ОПП как одной из составляющих обеспечения качественного образовательного проекта в динамической системе подготовки специалистов в области ИБ. Предложена структурная схема проектно-образовательной среды ОПП, которая наглядно демонстрирует взаимодействие циклов дисциплин и компетенций, формирующиеся в процессе обучения. Разработана математическая модель требований к ОКХ и ОПП в соответствии с условиями образовательного проекта подготовки специалистов в области ИБ, дающая возможность сотрудникам ВУЗ ГСЧС Украины компонировать сбалансированные циклы дисциплин для формирования в учащихся затребованных компетенций.
\end{abstract}

\footnotetext{
Львовский государственный университет безопасности жизнедеятельности; zahar_stashevsky@ukr.net / Lviv State University of Life Safety, Ukraine;

2 Национальный университет „Львовская политехника” / Lviv Polytechnic National University, Ukraine;

3 Авторы внесли одинаковый вклад в создание статьи / The authors contributed equally to this article;
} 
Ключевые слова: проектно-образовательная среда, информационная система (ИС), компетенция, компетентностный подход, образовательно-профессиональная программа

Вид статьи: оригинальная научная статья

\section{A B S T R A C T}

Aim: Analysis and identification of the main components relevant to the Information Security (IS) training structure for specialists undergoing Bachelor degree studies at educational institutions with special training conditions. These are key links in the pursuit of quality in the educational process. Additionally, the development of a mathematical model, incorporating student educational qualification profiles (EQP) and Vocation Development Programmes (VDP), will allow for the creation of suitable education programmes aimed at securing competences required by employers.

Introduction: Automated management systems introduced for the State Security Emergency Services (SSES) of Ukraine are designed for data collection, processing and transfer of large volumes of information between all emergency services. Such systems ensure timely, orderly and smooth functioning of state services dealing with emergency situations. However, just like any other Information System, this information should be protected against the possibility of intrusion and impact of cyber criminal activity. From the authors' analysis of threat sources and information system weaknesses it is evident that the human factor and staff training are critical elements in the establishment of good quality and efficient performance of the IS systems in SSES of Ukraine. To address identified problems, it is necessary to create a flexible departmental system of specialist training in the field of IS through the support of higher educational institution of SSES of Ukraine with special training conditions. This approach is based on principles for modern higher education in Ukraine, realized under the auspices of the "Bologna Declaration for higher vocational education in Ukraine". It uses a competency based approach, involving a close interaction between the employment market and education institutions.

Conclusions: A structural analysis was performed and key assumptions identified for the development of vocational training. This facilitated the production of an effective and dynamic education programme, to address the needs of IS. A project scheme was proposed, which visualised an interaction between the vocation education cycle and competences emerging during the training process. A mathematical "needs" model was developed, which revealed student profiles and a vocation development programme, in accordance with the education project conditions concerned with preparing specialists for work with IS. Simultaneously, this approach afforded staff from higher education establishments an opportunity to prepare a suitable syllabus, to address student competence needs.

Keywords: design and educational environment, information system, competence, competence approach, educational and professional program

Type of article: original scientific article

\section{A B S T R A K T}

Cel pracy: Analiza i identyfikacja głównych elementów struktury programu kształcenia specjalistów ds. bezpieczeństwa informacji na studiach licencjackich w uczelniach ze specjalnymi warunkami kształcenia, który jest jednym z ogniw zapewnienia jakości procesu kształcenia. Opracowanie modelu matematycznego wymagań dotyczących charakterystyki profilu absolwenta oraz programu edukacyjno-zawodowego, które dadzą możliwość stworzenia zrównoważonych programów nauczania mających na celu zdobycie przez absolwenta wyższej uczelni kompetencji wymaganych przez pracodawców.

Wprowadzenie: Dostępne dzisiaj zautomatyzowane systemy zarządzania w strukturach Państwowej Służby Ukrainy ds. Sytuacji Nadzwyczajnych są przeznaczone do gromadzenia danych oraz przetwarzania i przekazywania pomiędzy wszystkimi służbami ratowniczymi dużych ilości informacji. Dzięki temu służba działa terminowo, w sposób uporządkowany i bez zakłóceń. Jednak tak jak każdy system informacyjny, powinien być on chroniony przed działaniami cyberprzestępców. Z przeprowadzonej przez autorów analizy źródeł zagrożeń i słabości systemu informacyjnego wynika, że czynnik ludzki i poziom szkolenia personelu są niezbędne do stworzenia systemu dobrej jakości i sprawnego funkcjonowania systemów ochrony informacji Państwowej Służby Ukrainy ds. Sytuacji Nadzwyczajnych .

Aby rozwiązać przedstawione problemy, konieczne jest stworzenie odpowiedniego, elastycznego resortowego systemu przygotowania specjalistów z zakresu ochrony informacji przy wsparciu wyższych uczelni Państwowej Służby Ukrainy ds. Sytuacji Nadzwyczajnych ze specjalnymi warunkami kształcenia. System ten bazuje na zasadach nowoczesnego szkolnictwa wyższego Ukrainy, realizowanego w ramach „Deklaracji Bolońskiej w szkolnictwie wyższym zawodowym Ukrainy”, na podejściu opartym na kompetencjach, zakładającym ścisłą zależność między rynkiem pracy i resortowymi instytucjami edukacyjnymi. Wnioski: Przeprowadzono analizę struktury i określono główne założenia na potrzeby opracowania programu kształcenia zawodowego jako ogniwa w procesie przygotowania efektywnego projektu edukacyjnego w dynamicznym systemie kształcenia specjalistów ds. bezpieczeństwa informacji. Zaproponowany został schemat środowiska projektu edukacyjnego, który wizualizuje zależności cykli nauczania i kompetencji, powstałych w procesie nauki. Opracowany został model matematyczny wymagań odnośnie profilu absolwenta oraz programu kształcenia zawodowego zgodnie z warunkami projektu edukacyjnego 
przygotowania specjalistów ds. bezpieczeństwa informacji, dający pracownikom uczelni wyższych Państwowej Służby Ukrainy ds. Sytuacji Nadzwyczajnych możliwość przygotowywania odpowiednich programów nauczania w celu uzyskania przez studentów wymaganych kompetencji.

Słowa kluczowe: projektowane środowisko edukacyjne, system informacyjny, kompetencja, podejście kompetencyjne, program kształcenia zawodowego

Typ artykułu: oryginalny artykuł naukowy

\section{1. Введение}

Наличие большого количества информационно-коммуникационных систем и компьютерных сетей, разрабатывающихся и внедряющихся в ГСЧС Украины, направлены на автоматизацию выполнения работ и уменьшения времени реагирования на чрезвычайные ситуации (далее ЧС), которые значительно влияют на жизнеобеспечение человека, общества и государства. Имеющиеся на сегодня АСУ в структурных подразделениях ГСЧС Украины обрабатывают большой объем информации, обеспечивают ее хранение и передачу между всеми звеньями спасательной службы, результатом которой является своевременная, сбалансированная и бесперебойная ее работа $[1,5]$. Но, как и любая информационная система (ИС), принимая во внимание проявление злоумышленников в информационной среде, она должна быть защищена от них. Из проведенного нами анализа источников угроз и уязвимостей ИС [8] видно, что человеческий фактор и уровень подготовки персонала имеют ключевое значение при качественном построении и бесперебойном функционировании систем защиты информации в ГСЧС Украины.

Для решения указанных проблем необходимо задействовать немалый профессионализм кадров, который, в свою очередь, предусматривает создание соответствующей ведомственной гибкой системы подготовки специалистов в области ИБ. Эта система базируется на принципах современного высшего образования, реализованных в положении „Болонская декларация в системе высшего профессионально-технического образования Украины" на основе компетентностного подхода, предусматривающего тесное взаимодействие когнитивного рынка трудоустройства и ведомственных учебных заведений.

\section{2. Анализ направлений модернизации образовательных проектов подготовки специалистов в области информационной безопасности}

На протяжении последнего десятилетия теоретики и практики высшего образования ведут дискуссии относительно потребности формирования у выпускников не только определенных знаний и умений, но и особых компетенций, ориентированных на способность применения их на практике, в реальной ситуации, при создании новой конкурентной продукции, в разных жизненных ситуациях и т.п. В результате этого возникает потребность внедрения такой системы образования, которая обеспечивает обретение учащимся комплекса компетенций, которые должны содержать: фундаментальные и технические знания; умение анализировать и решать проблемы с использованием междисциплинарного подхода; навыки применения методов проектного менеджмента; готовность к коммуникабельности и командной работе (П. Эерэр, Б. Хефер, Б. Креуси, С. Енемарк) $[6,7,9,10]$. Основные направления модернизации имеющегося образования в Украине определены в некоторых государственных документах, в частности, в Указе Президента Украины от 25 июня 2013 г., №344/2013 «Национальная стратегия развития образования в Украине на 2012-2021 годы» и плане действий Министерства образования и науки Украины для реализации распоряжения Кабинета Министров Украины от 17.11.2007 г., № 886-р. „Об одобрении плана мер, направленных на удовлетворение потребности рынка труда в квалифицированных рабочих кадрах", утвержденный приказом МОН Украины от 12.12.2007 г., №1121.

Понятно, что современная экономика Украины ориентирована на персонал, компетенции которых намного превосходят образовательные показатели большинства выпускников ВУЗов, в том числе и в области ИБ. Очевидно и то, что значимыми и более эффективными для успешной профессионально-технической деятельности является не отдельные знания выпускника, а обобщенные умения, проявляющиеся в способности решать жизненно важные и профессиональные задачи, способности к иноязычному общению, подготовка в области ИТ, ТЗИ, КСЗИ и др. Поэтому, компетентностный подход на сегодня затребован, прежде всего, производителями и предпринимателями, поэтому современная система 
высшего образования требует существенной модернизации [4].

Суть концептуальных проблем реализации компетентностного подхода определяется значительным количеством и разнообразием интересов всех субъектов, принимающих участие в этом процессе. Так, например, государство имеет опыт разработки образовательно-квалификационных характеристик (ОКХ) - четких перечней знаний и умений, значимых с точки зрения получения диплома о государственной итоговой аттестации [11]. Вместе с тем, для работодателя (например, подразделения ГСЧС Украины) большое значение имеют базовые коммуникативные и информационные компетенции, а также - наличие опыта работы в сфере ИБ определенной организации. Тем не менее, выпускники, учитывая современные образовательные тенденции, в основном ориентируются на престижность соответствующего диплома и на возможность продолжения образования, в том числе и за границей. Именно поэтому социальноличностные, общенаучные, инструментальные и профессиональные (обще-профессиональные, специализировано-профессиональные) компетенции не только отличаются по своей наполненности, но и связаны с потребностями разных субъектов образовательно-производственной деятельности [2]. Итак, для объективного оценивания имеющихся компетенций, которыми должны владеть выпускники ВУЗов, нужно разработать разные по смыслу и структуре диагностические процедуры с использованием современных АСУ.

Одним из основных нормативных документов отраслевого стандарта высшего образования Украины (ОСВОУ) является образовательно-профессиональная программа (ОПП), в которой определяется нормативный термин и содержание процесса обучения, нормативные формы государственной аттестации, устанавливаются требования к содержанию, объему и уровню образования, а также профессиональной подготовки специалиста образовательно-квалификационных уровней не только бакалавра, но и специалиста и магистра по таким специальностям:

Таблица 1. Направления подготовки и специальности в область знаний 1701 ИБ

\begin{tabular}{|c|c|c|}
\hline Область знаний & Направления подготовки & $\begin{array}{l}\text { Название специальностей } \\
\text { специалиста и магистра }\end{array}$ \\
\hline \multirow{5}{*}{$\begin{array}{l}1701 \\
\text { информационная } \\
\text { безопасность }\end{array}$} & \multirow{2}{*}{$\begin{array}{c}6.170101 \text { - безопасность } \\
\text { информационных и } \\
\text { коммуникационных систем }\end{array}$} & $\begin{array}{c}\text { 7(8).17010101 - безопасность информационных } \\
\text { и коммуникационных систем }\end{array}$ \\
\hline & & $\begin{array}{c}\text { 7(8).17010102 - безопасность государственных } \\
\text { информационных ресурсов }\end{array}$ \\
\hline & $\begin{array}{c}6.170102 \text { - системы технической } \\
\text { защиты информации }\end{array}$ & $\begin{array}{c}\text { 7(8).17010201 - системы технической защиты информации, } \\
\text { автоматизация ее обработки }\end{array}$ \\
\hline & \multirow{2}{*}{$\begin{array}{c}6.170103 \text { - управление } \\
\text { информационной безопасностью }\end{array}$} & $\begin{array}{c}\text { 7(8).17010301 - управленаие информационной } \\
\text { безопасностью }\end{array}$ \\
\hline & & $\begin{array}{c}\text { 7(8).17010302 - административный менеджмент в сфере } \\
\text { защиты информации }\end{array}$ \\
\hline
\end{tabular}

Источник: Собственное исследование.

Table 1. Areas of training and specialization in the field of knowledge 1701 IS

\begin{tabular}{|c|c|c|}
\hline $\begin{array}{c}\text { Area of } \\
\text { expertise }\end{array}$ & Areas of training & Specialist's and master's specialisations \\
\hline \multirow{5}{*}{$\begin{array}{l}1701 \\
\text { information } \\
\text { security }\end{array}$} & \multirow{2}{*}{$\begin{array}{l}6.170101 \text { - security of informational and } \\
\text { communicational systems }\end{array}$} & $\begin{array}{c}7(8) .17010101 \text { - security of informational and communicational } \\
\text { systems }\end{array}$ \\
\hline & & 7(8).17010102 - security of state information resources \\
\hline & $\begin{array}{c}6.170102 \text { - system of technical protection } \\
\text { of information }\end{array}$ & $\begin{array}{c}\text { 7(8).17010201 - technical protection of information system, } \\
\text { automatization of its processing }\end{array}$ \\
\hline & \multirow{2}{*}{$\begin{array}{l}6.170103 \text { - management of informational } \\
\text { security }\end{array}$} & 7(8).17010301 - management of informational security \\
\hline & & $\begin{array}{c}\text { 7(8).17010302 - administrative management in the field of } \\
\text { information security }\end{array}$ \\
\hline
\end{tabular}

Source: Own elaboration.

Структура и последовательность этапов разработки ОПП согласовываются с формой ОКХ, являющиеся нормативным документом ОСВОУ. Структура ОКХ и ОПП и ее обязательное 
содержание являются стандартными. Рассмотрим к примеру более детально содержание и структуру ОПП.

Во вступлении содержится информация относительно использования ОПП при:

- разработке составляющей ОСВОУ, а также средства диагностики качества высшего образования (СДКВО);

- разработке составляющих ОСВОУ ВУЗа (вариативные части ОПП подготовки специалистов и СДКВО);

- планировании и организации учебного процесса (в частности, разработка учебного плана, програм учебных дисциплин и практик, строка обучения и т.п.);

- определении содержания процесса обучения в системе переподготовки и повышения квалификации;

- профессиональной ориентации соискателей соответствующей специальности;

- лицензировании и аккредитации, инспектировании образовательной деятельности по направлению подготовки и специальности.

- ОПП устанавливает:

- нормативный срок обучения по дневной форме;

- нормативные формы государственной атестации;

- нормативную часть содержания процесса обучения в зачетных единицах, усвоение которых обеспечивает формирование компетенций согласно требованиям ОКХ;

- перечень учебных дисциплин и практик.

Право на реализацию ОПП имеют ВУЗы в случае наличия соответствующей лицензии, выданной уполномоченным органом исполнительной власти.

Содержание раздела „Нормативные ссылки” является обязательным для каждой ОПП, который дополняется разработчиками, если там указываются ссылки на любые другие нормативные документы.

В разделе „Определение” приведены термины и соответствующие определения, представленные в «Комплексе нормативных документов для разработки составных системы стандартов высшего образования». В случае необходимости, раздел дополняется разработчиками ОПП.

В разделе „Обозначение и сокращение” приведено сокращенное название циклов подготовки, применяющиеся при формировании шифров компетенций, а именно:

- ГСЭ (01) - гуманитарной и социально-экономической подготовки;
- $\mathrm{MEH}(02)$ - математической, естественно-научной подготовки;

- ПП (03) - профессиональной и практической подготовки.

В разделе „Распределение содержания процесса обучения и учебного времени по циклам подготовки, учебными дисциплинами и практиками” приводится распределение содержания ОПП специалиста и учебное время по нормативной и вариативной части, учебное время по циклам подготовки, количество учебных часов/кредитов изучения каждой из учебных дисциплин и практик нормативной части программы подготовки, которые сводятся в соответствующую таблицу.

В разделе „Нормативная часть содержания образовательно-профессиональной программы” указываются:

- распределение содержания ОПП и учебного времени по циклам подготовки, которые сведены в соответствующую таблицу;

- система знаний в виде системы содержательных модулей, которые сведены в соответствующую таблицу;

- перечень нормативных учебных дисциплин и практик (видов практической подготовки), где указываются названия и шифры блоков содержательных модулей, из которых формируются эти дисциплины и практики, и названия и шифры содержательных модулей, входящие в определенный блок содержательных модулей, которые также сводятся в соответствующую таблицу.

На завершающем этапе разработки ОПП создается соответствующая таблица, в которой для каждой учебной дисциплины (или практики) нормативной части содержания ОПП указывается количество учебных часов/ национальных кредитов/ кредитов ECTS ее изучение и перечень сформированных компетенций. Однако ВУЗ имеет право менять названия учебных дисциплин и практик и распределение блоков содержательных модулей в них при отдельном согласовании с МОН Украины.

Структуру ОПП специалиста образовательноквалификационного уровня бакалавр по направлению подготовки 6.170103 „Управление информационной безопасностью” можно представить в виде ментальных карт для удобства и наглядности пользования (см. рис. 1). На этом рисунке введены такие обозначения:

$\stackrel{\tilde{K}}{=}=\left\{\tilde{K}_{i}=\left\{k_{i j}, j=\overline{1, n_{i}}\right\}, i=\overline{1, m^{d}}\right\}$ - i-тая компетенция, формирующаяся $j$-той дисциплиной; $\tilde{D}=\left\{d_{i}, i=1, m^{d}\right\}-i$-тая дисциплина, формирующая определенную компетенцию. 


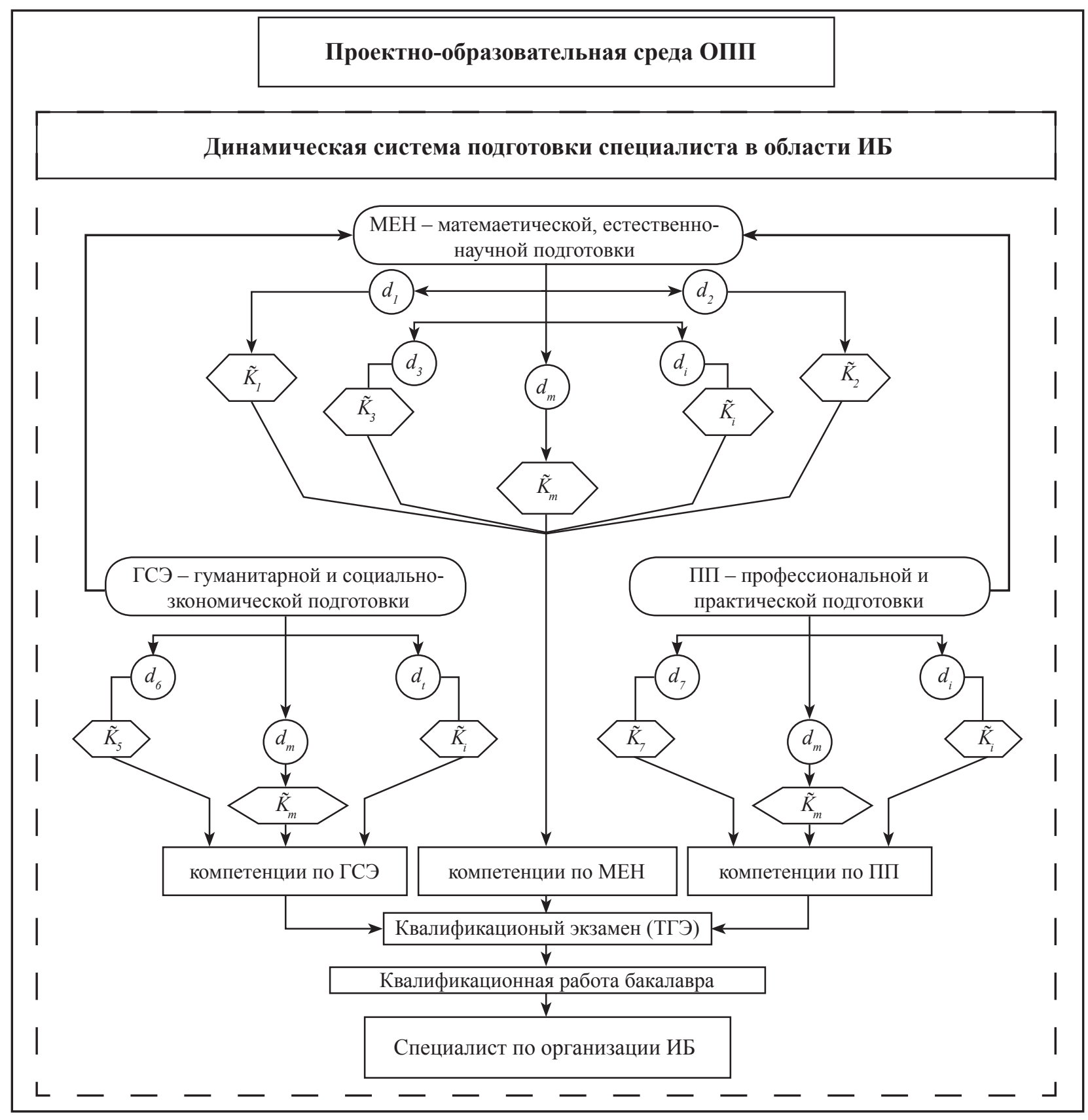

Рис. 1. Структурная схема проектно-образовательной среды ОПП в динамической системе подготовки специалиста в области ИБ

Источник: Собственное исследование. 


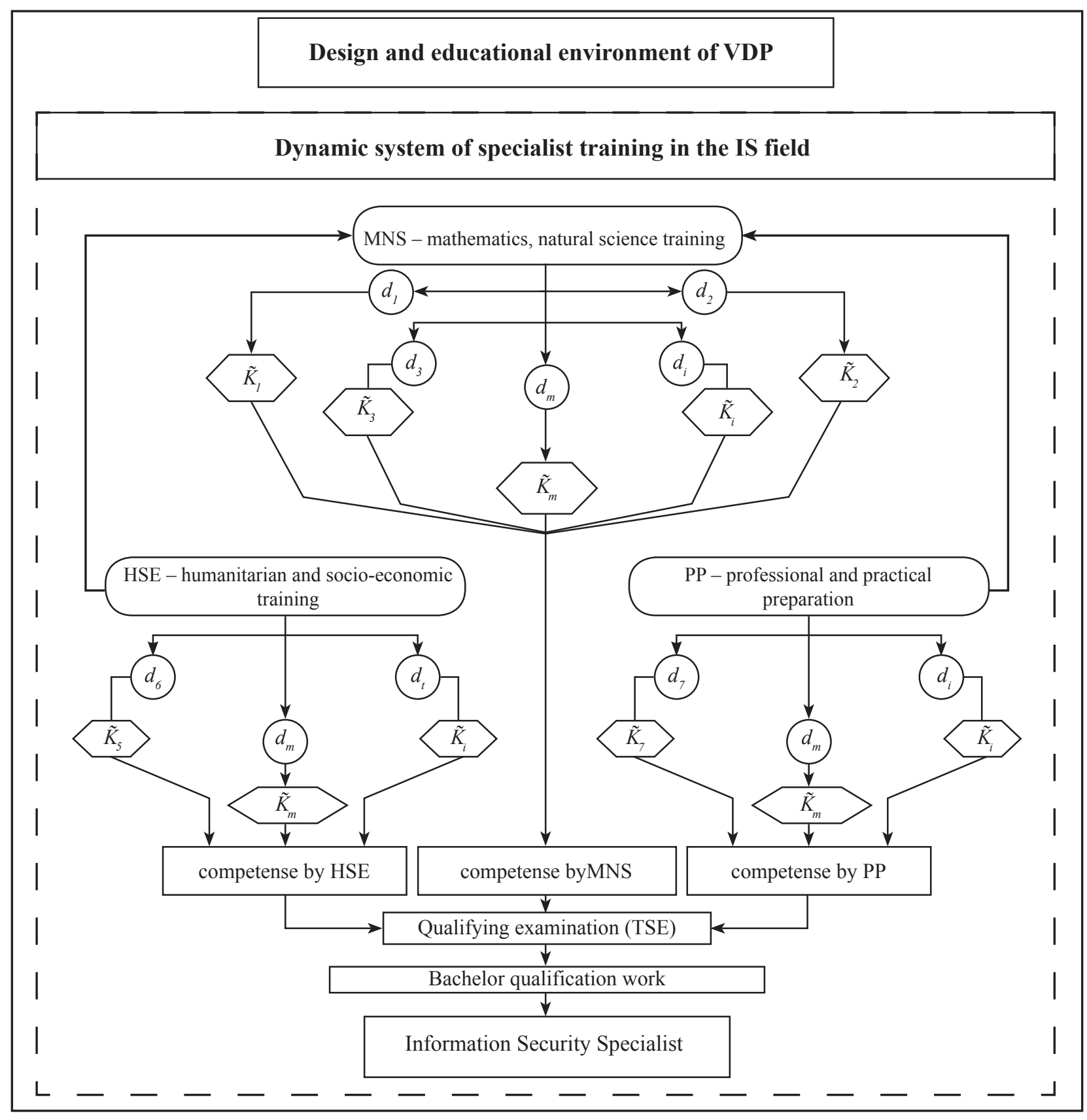

Fig. 1. Structural scheme of design and educational environment Vocation Development Programmes (VDP) in a dynamic system of specialist training in the IS field

Source: Own elaboration.

Таким образом, разработка и соблюдение ОПП является важным фактором организации учебного процесса в области ИБ в ВУЗах, находящихся в системе ГСЧС Украины, одной из предпосылок подготовки высококвалифицированных специалистов для подразделений управления телекоммуникациями, информационных технологий и Системы 112, сектора специальной связи и режимно-секретных отделов при главных управлениях ГСЧС Украины.

\section{3. Математическая модель образовательного проекта подготовки специалиста в области ИБ}

Принимая во внимание сказанное выше и следуя системному подходу [3] структуру требований к составным ОСВОУ представим в виде трех компонент: 1) учебные циклы; 2) дисциплины; 3) знание, умение и навыки. Эти компоненты являются взаимосвязанными: учебные циклы определяются набором дисциплин, которые, представляются в виде совокупности знаний, умений и навыков. 
Пусть ОПП ОСВОУ описывается таким множеством свойств

$\tilde{Y}=\{\tilde{Z}, \tilde{U}, \tilde{S}\}$

где: $\tilde{Z}=\left\{z_{i}, i=\overline{1, m^{z}}\right\}$ - множество знаний;

$\tilde{U}=\left\{u_{i}, i=\overline{1, m^{\mathrm{u}}}\right\}$ - множество умений;

$\tilde{S}=\left\{s_{i}, i=\overline{1, m^{\mathrm{s}}}\right\}-$ множество навыков, причем

$\tilde{Y}=\tilde{Z} \cup \tilde{U} \cup \tilde{S}, \tilde{Z} \cap \tilde{U}=\varnothing, \tilde{Z} \cap \tilde{S}=\varnothing$,

$\tilde{U} \cap \tilde{S}=\varnothing$.

Это означает, что каждый элемент этого множества влияет на общие свойства ОСВОУ. Степень влияния элементов реализуется через соответствующее множество дисциплин

$\tilde{D}=\left\{d_{i}, i=\overline{1, m^{\mathrm{d}}}\right\}$

то есть в общем случае это влияние будет иметь такой вид:

$P: \tilde{D} \rightarrow \tilde{Y}, p(d)=\tilde{Y}^{\prime} \Leftrightarrow \tilde{Y}$

причем, результаты влияния должны полностью отвечать свойствам ОПП.

Поскольку ОСВОУ базируется на принципах компетентностного подхода к высшему профессионально-техническому образованию, то по результатам освоения ОПП в области ИБ выпускник должен владеть набором определенных компетенций. Для любого ОСВОУ определенна компетентносная структура содержания процесса образования, наполненная требованиями к результатам образовательной деятельности по направлению подготовки, определенному ОСВОУ в области ИБ.

В ОСВОУ образовательно-квалификационного уровня бакалавр используются такие виды компетенций (см. прил. А с ОКХ): КСЛ - социальноличностные; КОН - общенаучные; КИ - инструментальные; КОП - обще-профессиональные; КСП - специализированно-профессиональные.

Определим для ОКХ ОСВОУ такое множество компетенций:

$\tilde{\tilde{G}}^{\mathrm{K}}=\left\{\tilde{G}_{i}^{\mathrm{K}}=\left\{g_{i j}^{\mathrm{K}}, j=\overline{1, n_{i}^{\mathrm{K}}}\right\}, i=\overline{1, m^{\mathrm{K}}}\right\}$

разделенных на соответствующие группы, а именно $\tilde{\tilde{G}}^{\mathrm{K}}=\bigcup_{i=1}^{m^{\mathrm{K}}} \tilde{G}_{i}^{\mathrm{K}}: \tilde{G}_{i}^{\mathrm{K}} \cap \tilde{G}_{j}^{\mathrm{K}}=\varnothing, i \neq j: \forall i, \forall j \in m^{\mathrm{K}}$

где:

- $\tilde{G}_{1}^{\mathrm{K}}=\left\{g_{1, j}^{\mathrm{K}}, j=\overline{1, n_{1}^{\mathrm{K}}}\right\}-$ КСЛ (компетенции социально-личностные), $n_{1}^{\mathrm{K}}=10$;

$\tilde{G}_{2}^{\mathrm{\kappa}}=\left\{g_{2, j}^{\mathrm{K}}, j=\overline{1, n_{2}^{\mathrm{K}}}\right\}-$ КОН (компетенции общенаучные), $n_{2}^{\mathrm{\kappa}}=5$;
- $\tilde{G}_{3}^{\mathrm{K}}=\left\{g_{3, j}^{\mathrm{\kappa}}, j=\overline{1, n_{3}^{\mathrm{\kappa}}}\right\}-$ КИ (компетенции инструментальные), $n_{3}^{\mathrm{K}}=5$;

- $\tilde{G}_{4}^{\mathrm{K}}=\left\{g_{4, j}^{\mathrm{K}}, j=\overline{1, n_{4}^{\mathrm{K}}}\right\}-$ КОП (компетенции обще-профессиональные), $n_{4}^{\mathrm{K}}=21$;

- $\tilde{G}_{5}^{\mathrm{\kappa}}=\left\{g_{5, j}^{\mathrm{K}}, j=\overline{1, n_{5}^{\mathrm{\kappa}}}\right\}-$ КСП (компетенции специализировано-профессиональные), $n_{5}^{\mathrm{K}}=19$;

- $g_{i j}^{\text {к }}$ - значение $j$-ого элемента для $i$-той группы компетенций (см. прил. А с ОКХ) [12].

Выражение (4) отвечает такому шифру компетенции:

\begin{tabular}{|l|l} 
KXX $\quad$\begin{tabular}{l} 
XX $\quad$ номер (элемент) компетенции \\
\cline { 2 - 2 }
\end{tabular} \\
аббревиатура компетенции
\end{tabular}

\begin{tabular}{|ll} 
KXX $\quad\lfloor X \quad$ number (element) of competence \\
\cline { 2 - 2 }
\end{tabular}

Например, шифр КСЛ-06 означает социальноличностная компетенция (КСЛ) $\rightarrow$ адаптивность и коммуникабельность (06); КСП-07 - специализировано-профессиональная компетенция (КСП) $\rightarrow$ способность использовать профессионально профилированные знания в области ИБ для проектирования угроз ИБ (07).

В ОСВОУ образовательно-квалификационного уровня бакалавр используются такие виды умений: ПП - предметно-практическое; ПУ - предметно-умственние; ЗП - знаково-практические; ЗУ - знаково-умственние. Вместе с тем, каждому виду умений отвечают разные уровни сформированности умения: О - способность выполнять действие, опираясь на материальные носители информации относительно нее; Р - способность выполнять действие, опираясь на постоянный умственный контроль без помощи материальных носителей информации; $\mathrm{H}$ - способность выполнять действие автоматически, на уровне привычки.

Определим для ОКХ ОСВОУ такое множество видов умений:

$$
\tilde{\tilde{U}}^{\mathrm{y}}=\left\{\tilde{U}_{i}^{\mathrm{y}}=\left\{u_{i j}^{\mathrm{y}}, j=\overline{1, n_{i}^{\mathrm{y}}}\right\}, i=\overline{1, m^{\mathrm{y}}}\right\}
$$

разделенных на соответствующие уровни сформированности умения

$\tilde{\tilde{U}}^{\mathrm{y}}=\bigcup_{i=1}^{m^{\mathrm{y}}} \tilde{U}_{i}^{\mathrm{y}}: \tilde{U}_{i}^{\mathrm{y}} \cap \tilde{U}_{j}^{\mathrm{y}}=\varnothing, i \neq j: \forall i, \forall j \in m^{\mathrm{y}}$

где:

- $\tilde{U}_{1}^{\mathrm{y}}=\left\{u_{1, j}^{\mathrm{y}}, j=\overline{1, n_{1}^{\mathrm{y}}}\right\}-$ ПП (предметно-практическое), $n_{1}^{\mathrm{y}} \in\{\mathrm{O}, \mathrm{P}, \mathrm{H}\}$; 
- $\tilde{U}_{2}^{\mathrm{y}}=\left\{u_{2, j}^{\mathrm{y}}, j=\overline{1, n_{2}^{\mathrm{y}}}\right\}-$ ПУ (предметно-умственное), $n_{2}^{\mathrm{y}} \in\{\mathrm{O}, \mathrm{P}, \mathrm{H}\}$;

- $\tilde{U}_{3}^{\mathrm{y}}=\left\{u_{3, j}^{\mathrm{y}}, j=\overline{1, n_{3}^{\mathrm{y}}}\right\}-3 П$ (знаково-практическое), $n_{3}^{\mathrm{y}} \in\{\mathrm{O}, \mathrm{P}, \mathrm{H}\}$;

- $\tilde{U}_{4}^{\mathrm{y}}=\left\{u_{4, j}^{\mathrm{y}}, j=\overline{1, n_{4}^{\mathrm{y}}}\right\}-3 \mathrm{У}$ (знаково-умственное), $n_{4}^{\mathrm{y}} \in\{\mathrm{O}, \mathrm{P}, \mathrm{H}\}$;

- $u_{i j}^{\mathrm{y}}$ - значение $j$-ого уровня сформированности умения для $i$-ого вида умений (см. прил. В с OKX) [12].

Согласно ОКХ ОСВОУ, $j$-ому элементу для $i$-той группы компетенций выпускника ВУЗа отвечает определенная система умений, отображающие их, а также соответствующие уровни сформированности умения:

$$
\begin{aligned}
\tilde{\tilde{G}}^{\mathrm{K}}=\left\{\tilde{G}_{i}^{\mathrm{K}}=\left\{P: g_{i j}^{\mathrm{K}}\right.\right. & \rightarrow \bigcup_{k \in m^{\mathrm{y}}} \bigcup_{l \in n_{k}^{\mathrm{y}}} u_{k l}^{\mathrm{y}}, j= \\
& \left.\left.=\overline{1, n_{i}^{\mathrm{K}}}\right\}, i=\overline{1, m^{\mathrm{K}}}\right\}
\end{aligned}
$$

причем

$u_{k l}^{\mathrm{y}} \cap u_{k t}^{\mathrm{y}}=\varnothing, l \neq t: \forall l, \forall t \in n_{k}^{\mathrm{y}} ; \forall k \in m^{\mathrm{K}}$

\begin{tabular}{|c|c|c|c|c|}
\hline \multirow[t]{4}{*}{ KXX-XX } & \multirow[t]{2}{*}{ XX } & \multirow[t]{2}{*}{$\mathrm{X}$} & $\mathrm{XX}$ & $\begin{array}{l}\text { номер умения, сквозной для } \\
\text { этой компетенции }\end{array}$ \\
\hline & & & & $\begin{array}{l}\text { уровень сформированности } \\
\text { умения }\end{array}$ \\
\hline & & & & вид умения \\
\hline & & & & шифр компетенции \\
\hline
\end{tabular}

Выражение (7) отвечает такому шифру умения:

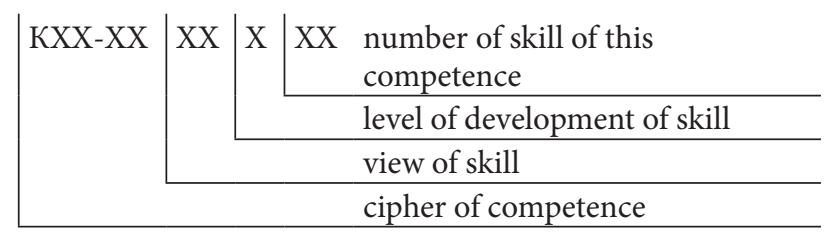

Например, шифр КСЛ-06.ПП.О.02 означает, что выпускник ВУЗа должны иметь социально-личностную компетенцию (КСЛ), элементом которой является адаптивность и коммуникабельность (06), их отображает предметно-практический вид умения (ПП), уровень сформированности которого обеспечивает способность выполнять действие, опираясь на материальные носители информации относительно нее (O), при этом выпускник должен творчески использовать современные технологии связи с общественностью для оперативного и эффективного решения управленческих задач (02).
В ОСВОУ образовательно-квалификационного уровня бакалавр используются такие производственные функции (см. прил. Б. с ОКХ): проектная; прогностическая; исследовательская; организационная; техническая; технологическая; управленческая; контрольная. Определим для ОКХ ОСВОУ такое множество производственных функций:

$\tilde{\tilde{Q}}^{\phi}=\left\{\tilde{Q}_{i}^{\phi}=\left\{q_{i j}^{\phi}, j=\overline{1, n_{i}^{\phi}}\right\}, i=\overline{1, m^{\phi}}\right\}$

разделенных на соответствующие типичные задачи деятельности, а именно

$\tilde{\tilde{Q}}^{\phi}=\bigcup_{i=1}^{m^{\mathrm{K}}} \tilde{Q}_{i}^{\phi}: \tilde{Q}_{i}^{\phi} \cap \tilde{Q}_{j}^{\phi}=\varnothing, i \neq j: \forall i, \forall j \in m^{\phi}$

где: $\tilde{Q}_{1}^{\phi}=\left\{q_{1, j}^{\phi}, j=\overline{1, n_{1}^{\phi}}\right\}$ - проектная, $n_{1}^{\phi}=3$;

- $\tilde{Q}_{2}^{\phi}=\left\{q_{2, j}^{\phi}, j=\overline{1, n_{2}^{\phi}}\right\}$ - прогностическая, $n_{2}^{\phi}=2$;

- $\tilde{Q}_{3}^{\phi}=\left\{q_{3, j}^{\phi}, j=\overline{1, n_{3}^{\phi}}\right\}$ - исследовательская, $n_{3}^{\phi}=1$;

- $\tilde{Q}_{4}^{\phi}=\left\{q_{4, j}^{\phi}, j=\overline{1, n_{4}^{\phi}}\right\}$ - организационная, $n_{4}^{\phi}=4$;

- $\tilde{Q}_{5}^{\phi}=\left\{q_{5, j}^{\phi}, j=\overline{1, n_{5}^{\phi}}\right\}$ - техническая, $n_{5}^{\phi}=2$;

- $\tilde{Q}_{6}^{\phi}=\left\{q_{6, j}^{\phi}, j=\overline{1, n_{6}^{\phi}}\right\}$ - технологическая, $n_{6}^{\phi}=2$;

- $\tilde{Q}_{7}^{\phi}=\left\{q_{7, j}^{\phi}, j=\overline{1, n_{7}^{\phi}}\right\}$ - управленческая, $n_{7}^{\phi}=5$;

- $\tilde{Q}_{8}^{\phi}=\left\{q_{8, j}^{\phi}, j=\overline{1, n_{8}^{\phi}}\right\}$ - контрольная, $n_{8}^{\phi}=2$.

- $q_{i j}^{\phi}$ - значение j-ой типичной задачи деятельности для $i$-той производственной функции (см. прил. Б. с ОКХ) [12].

В ОСВОУ образовательно-квалификационного уровня бакалавр используются такие виды типичных задач деятельности: ПФ - профессиональный; СП - социально-производственный; СБ - социально-бытовой. Вместе с тем, каждому виду типичной задачи деятельности отвечают разные классы задач деятельности: С - стереотипный; Д - диагностический; Э - эвристический.

Определим для ОКХ ОСВОУ такое множество видов типичных задач деятельности:

$\tilde{\tilde{Z}}^{\text {д }}=\left\{\tilde{Z}_{i}^{\text {д }}=\left\{z_{i j}^{\text {д }}, j=\overline{1, n_{i}^{\text {д }}}\right\}, i=\overline{1, m^{\text {д }}}\right\}$ разделенных на соответствующие классы задач деятельности, а именно 
$\tilde{\tilde{Z}}^{\text {д }}=\bigcup_{i=1}^{m^{\text {д }}} \tilde{Z}_{i}^{\text {д }}: \tilde{Z}_{i}^{\text {д }} \cap \tilde{Z}_{j}^{\text {д }}=\varnothing, i \neq j: \forall i, \forall j \in m^{\text {д }}$

где:

- $\tilde{Z}_{1}^{\text {д }}=\left\{z_{1, j}^{\text {д }}, j=\overline{1, n_{1}^{\text {д }}}\right\}-$ ПФ (профессиональный), $n_{1}^{\text {д }} \in\{\mathrm{C}$, Д, Е $\}$;

- $\tilde{Z}_{2}^{\text {д }}=\left\{z_{2, j}^{\text {д }}, j=\overline{1, n_{2}^{\text {д }}}\right\}-$ СП (социально-производственный), $n_{2}^{\text {д }} \in\{\mathrm{C}$, Д, $\mathrm{E}\}$;

- $\tilde{Z}_{3}^{\text {д }}=\left\{z_{3, j}^{\text {д }}, j=\overline{1, n_{3}^{\text {д }}}\right\}-$ СБ (социально-бытовой), $n_{3}^{\text {Д }} \in\{\mathrm{C}$, Д, $\mathrm{E}\}$;

- $z_{i j}^{\text {д }}$ - значение ј-ого класса задач деятельности для і-ого вида типичных задач деятельности (см. прил. Б. с ОКХ) [12].

Согласно ОКХ ОСВОУ, $j$-ому виду типичной задачи деятельности для $i$-той производственной функции, которыми должен владеть выпускник ВУЗа, отвечает определенный класс типичной задачи деятельности, отображающий их, а также номер задачи, сквозной для этой производственной функции:

$$
\begin{aligned}
& \tilde{\tilde{Q}}^{\phi}=\left\{\tilde{Q}_{i}^{\phi}=\left\{P: q_{i j}^{\phi} \rightarrow \bigcup_{k \in m^{\mathbb{A}} l \in n_{k}^{\mathbb{A}}} z_{k l}^{\mathrm{M}},\right.\right. \\
& \left.\left.j=\overline{1, n_{i}^{\phi}}\right\}, i=\overline{1, m^{\phi}}\right\}
\end{aligned}
$$

причем $z_{k l}^{\text {Д }} \cap z_{k t}^{\text {д }}=\varnothing, l \neq t: \forall l, \forall t \in n_{k}^{\text {д }} ; \forall k \in m^{\text {म }}$

Выражение (12) отвечает такому шифру типичной задачи деятельности:

$$
|\mathrm{XX}| \mathrm{XX}|\mathrm{X}| \begin{aligned}
& \mathrm{XX} \begin{array}{l}
\text { номер задачи, сквозной для этой } \\
\text { производственной функции }
\end{array} \\
& \begin{array}{l}
\text { класс типичной задачи деятельности } \\
\text { вид типичной задачи деятельности }
\end{array} \\
& \text { номер производственной функции }
\end{aligned}
$$

\begin{tabular}{|l|}
$\mathrm{XX}|\mathrm{XX}| \mathrm{X} \mid \mathrm{XX} \begin{array}{l}\text { tasks number of the production } \\
\text { function }\end{array}$ \\
$\frac{\text { class of typical action tasks }}{\text { view of typical action tasks }}$ \\
number of the production function
\end{tabular}

Например, шифр 1.ПФ.Е.01 означает, что выпускники ВУЗа должны иметь 1-вую производственную функцию (проектную), которой отвечает профессиональный вид типичной задачи деятельности (ПФ), их отображает эвристический класс типичной задачи деятельности (Е), при этом выпускник должен владеть навыками разработки программных документов относительно управления системой обеспечения информационной безопасности (01).

Согласно ОКХ ОСВОУ, $j$-ому виду типичной задачи деятельности для $i$-той производственной функции, которыми должен владеть выпускник ВУЗа, отвечает определенный класс и соответствующий номер типичной задачи деятельности, который их отображает, а также определенная система умений с соответствующими уровнями их сформированности и номером умения, сквозного

\begin{tabular}{|c|c|c|c|c|}
\hline \multirow[t]{4}{*}{ KXX-XX } & \multirow[t]{3}{*}{ XX } & $\mathrm{X}$ & XX & $\begin{array}{l}\text { номер умения, сквозной для } \\
\text { этой компетенции }\end{array}$ \\
\hline & & & & $\begin{array}{l}\text { уровень сформированности } \\
\text { умения }\end{array}$ \\
\hline & & & & вид умения \\
\hline & & & & шифр компетенции \\
\hline
\end{tabular}
для этой компетенции:

$$
\begin{array}{r}
\tilde{Q}^{\phi}=\left\{\tilde{Q}_{i}^{\phi}=\left\{P: q_{i j}^{\phi} \rightarrow \bigcup_{k \in m^{\mathbb{x}}} \bigcup_{l \in n_{k}^{M}} z_{k l}^{\text {ม }} \rightarrow\right.\right. \\
\left.\left.\rightarrow \bigcup_{m \in m^{\mathrm{y}}} \bigcup_{n \in n_{m}^{\mathrm{y}}} u_{m n}^{\mathrm{y}}, j=\overline{1, n_{i}^{\phi}}\right\}, i=\overline{1, m^{\phi}}\right\}
\end{array}
$$

где $z_{k l}^{\text {Д }} \cap z_{k t}^{\text {Д }}=\varnothing, l \neq t: \forall l, \forall t \in n_{k}^{\text {д }} ; \forall k \in m^{\text {д }}$, $u_{m n}^{\mathrm{y}} \cap u_{m p}^{\mathrm{y}}=\varnothing, n \neq p: \forall n, \forall p \in n_{m}^{\mathrm{y}} ; m \in m^{\mathrm{y}}$.

Выражение (13) отвечает такому шифру умения:

$\mid$\begin{tabular}{l|l} 
KXX-XX $|\mathrm{XX}| \mathrm{X} \mid \mathrm{XX} \begin{array}{l}\text { number of skill of this } \\
\text { competence }\end{array}$ \\
$\frac{\text { level of development of skill }}{\text { view of skill }}$
\end{tabular}

Например, шифр 1.ПФ.Е.01.ПУ.Г.01 означает, что выпускник ВУЗа должен иметь 1-вую производственную функцию (проектную), которой отвечает профессиональный вид типичной задачи деятельности (ПФ), их отображает эвристический класс типичной задачи деятельности (E), при этом выпускник, владея навыками разработки программных документов относительно управления системой обеспечения инбормационной безопасности (01), при уровне сформированности умения (Р), то есть способности выполнять действие, опираясь на постоянный умственный контроль без помощи материальных носителей информации, имеет в условиях профессиональной деятельности в составе группы специалистов или единолично согласно действующим нормативно-правовым актам готовить 
проекты нормативных документов (стратегии, программы, кониепиии, доктрины), исходя из всестороннего анализа и реальной оценки ситуации, ресурсного обеспечения и отбора адекватных механизмов внедрения управленческих решений (01).

Определим для ОПП ОСВОУ такое множество циклов подготовки:

$$
\begin{array}{r}
\tilde{\tilde{\widetilde{C}}}^{\Pi}=\left\{\tilde{\tilde{C}}_{i}^{\Pi}=\left\{\tilde{C}_{i j}^{\Pi}=\left\{c_{i j k}^{\Pi}, k=\overline{1, n_{i j}^{\Pi}}\right\},\right.\right. \\
\left.\left.j=\overline{1, \tilde{N}_{i}^{\Pi}}\right\}, i=\overline{1, m^{\Pi}}\right\}
\end{array}
$$

разделенных на соответствующие учебные дисциплины или практики:

$$
\tilde{\tilde{C}}^{\Pi}=\bigcup_{i=1}^{m^{\Pi}} \tilde{\tilde{C}}_{i}^{\Pi}: \tilde{\tilde{C}}_{i}^{\Pi} \bigcap \tilde{\tilde{C}}_{j}^{\Pi}=\varnothing, i \neq j: \forall i, \forall j \in m^{\Pi}
$$

каждая из которых состоит из разных блоков содержательных модулей

$$
\begin{array}{r}
\tilde{\tilde{\widetilde{C}}}^{\Pi}=\left\{\tilde{\tilde{C}}_{i}^{\Pi}=\bigcup_{j=1}^{\tilde{N}_{i}^{\Pi}} \tilde{C}_{i j}^{\Pi}: \tilde{C}_{i j}^{\Pi} \cap \tilde{C}_{i l}^{\Pi}=\varnothing,\right. \\
\left.j \neq l: \forall j, \forall l \in \tilde{N}_{i}^{\Pi}, i=\overline{1, m^{\Pi}}\right\}
\end{array}
$$

где:

- $\tilde{\tilde{C}}_{1}^{\Pi}=\left\{\tilde{C}_{1, j}^{\Pi}, j=\overline{1, \tilde{N}_{1}^{\Pi}}\right\}-$ ГСЕ (гуманитарной и социально-экономической подготовки), $\tilde{N}_{1}^{\text {д }}=10$;

- $\tilde{\tilde{C}}_{2}^{\text {п }}=\left\{\tilde{C}_{2, j}^{\text {п }}, j=\overline{1, \tilde{N}_{2}^{\Pi}}\right\}-\Pi H(\mathrm{MEH})$ (математической, естественно-научной подготовки), $\tilde{N}_{2}^{\text {д }}=3$;

- $\quad \tilde{\widetilde{C}}_{3}^{\Pi}=\left\{\tilde{C}_{3, j}^{\Pi}, j=\overline{1, \tilde{N}_{3}^{\Pi}}\right\}-\Pi \Pi$ (профессиональной и практической подготовки), $\tilde{N}_{3}^{\text {д }}=19+3$, где 3 - количество учебных практик;

- $c_{i j k}^{\Pi}$ - значение $k$-ого блока содержательных модулей $j$-той учебной дисциплины или практики для $i$-ого цикла подготовки (см. прил. В. с ОПП) [12].

Согласно ОПП ОСВОУ, $j$-той учебной дисциплины для $i$-ого цикла подготовки, которые должен усвоить выпускник ВУЗа, отвечает определенный блок содержательных модулей, отображающий их, а именно:

$$
\begin{array}{r}
\tilde{\tilde{C}}^{\Pi}=\left\{\tilde{\tilde{C}}_{i}^{\Pi}=\left\{P: \tilde{C}_{i j}^{\Pi} \rightarrow \bigcup_{k=1}^{n_{i j}^{\Pi}} c_{i j k}^{\Pi},\right.\right. \\
\left.\left.j=\overline{1, \tilde{N}_{i}^{\Pi}}\right\}, i=\overline{1, m^{\Pi}}\right\}
\end{array}
$$

причем $c_{i j k}^{\Pi} \cap c_{i j t}^{\Pi}=\varnothing, k \neq t: \forall k, \forall t \in n_{i j}^{\Pi}$;

$$
\forall j \in \tilde{N}_{i}^{\Pi} ; \forall i \in m^{\Pi}
$$

Выражение (17) отвечает такому шифру блока содержательных модулей, который входит в учебную дисциплину:

$|\mathrm{XX}| \mathrm{XX} \mid \begin{aligned} & \mathrm{XX} \\ & \begin{array}{l}\text { номер блока содержательного } \\ \text { модуля, сквозного для данного цикла } \\ \text { подготовки }\end{array} \\ & \text { номер учебной дисциплины }\end{aligned}$

$|\mathrm{XX}| \mathrm{XX} \mid \begin{aligned} & \mathrm{XX} \begin{array}{l}\text { block number of content modules of the } \\ \text { training cycle }\end{array} \\ & \text { number of discipline }\end{aligned}$

Например, шифр 1.01.01 означает: 1 - цикл гуманитарных и социально-экономических дисциплин (ГСЭ); 01 - номер учебной дисциплины ( $\rightarrow$ История Украины»; 01 - номер блока содержательного модуля $\rightarrow$ историческая деятельность и общественная жизнь украинского народа на разных этапах его существования.

В ОСВОУ применяются разные названия содержательных модулей, сквозные как для соответствующей компетенции, так и для соответствующего цикла подготовки. Определим для ОПП ОСВОУ такое множество содержательных модулей:

$\tilde{S}_{i}^{\Pi}=\left\{s_{i}^{\mathrm{M}}, i=\overline{1, m^{\mathrm{M}}}\right\}$

где $S_{i}^{\mathrm{M}}$ - название $i$-ого содержательного модуля (см. прил. Б. с ОПП) [12].

Согласно ОПП ОСВОУ, $j$-той учебной дисциплине для $i$-ого цикла подготовки, которые должны усвоить выпускники ВУЗа, отвечает определенный блок содержательных модулей, составляющими которого является некоторое множество содержательных модулей, а именно:

$$
\begin{array}{r}
\tilde{\tilde{C}}^{\Pi}=\left\{\tilde{\widetilde{C}}_{i}^{\Pi}=\left\{P: \tilde{C}_{i j}^{\Pi} \rightarrow \bigcup_{k=1}^{n_{i j}^{\Pi}} c_{i j k}^{\Pi} \rightarrow \bigcup_{p \in m^{\mathrm{M}}} s_{p}^{\mathrm{M}},\right.\right. \\
\left.\left.j=\overline{1, \tilde{N}_{i}^{\Pi}}\right\}, i=\overline{1, m^{\Pi}}\right\}
\end{array}
$$


где

$c_{i j k}^{\Pi} \cap c_{i j t}^{\Pi}=\varnothing, k \neq t: \forall k, \forall t \in n_{i j}^{\Pi} ; \forall j \in \tilde{N}_{i}^{\Pi} ;$

$\forall i \in m^{\mathrm{M}}, s_{v}^{\mathrm{M}} \cap s_{w}^{\mathrm{M}}=\varnothing, v \neq w: \forall v, \forall w \in m^{\mathrm{M}}$

Согласно ОПП ОСВОУ (см. прил. Б. и В), j-ому виду типичной задачи деятельности для $i$-той производственной функции, которыми должен владеть выпускник ВУЗа, отвечает определенный класс и соответствующий номер типичной задачи деятельности, который их отображает, а также определенная система умений с соответствующими уровнями их сформированности и номером умения, сквозного для этой компетенции, которым отвечает определенная система содержательных модулей:

$$
\begin{array}{r}
\tilde{\tilde{Q}}^{\Phi}=\left\{\tilde{Q}_{i}^{\phi}=\left\{P: q_{i j}^{\phi} \rightarrow \bigcup_{k \in m^{\text {д }}} \bigcup_{l \in n_{k}^{\mathrm{M}}} z_{k l}^{\text {д }} \rightarrow\right.\right. \\
\left.\left.\rightarrow \bigcup_{m \in m^{\mathrm{y}}} \bigcup_{n \in n_{m}^{\mathrm{y}}} u_{m n}^{\mathrm{y}} \rightarrow s_{v}^{\mathrm{M}}, j=\overline{1, n_{i}^{\phi}}\right\}, i=\overline{1, m^{\phi}}\right\}
\end{array}
$$

где $z_{k l}^{\text {д }} \cap z_{k t}^{\text {д }}=\varnothing, l \neq t: \forall l, \forall t \in n_{k}^{\text {д }} ; \forall k \in m^{\text {д }}$, $u_{m n}^{\mathrm{y}} \cap u_{m p}^{\mathrm{y}}=\varnothing, n \neq p: \forall n, \forall p \in n_{k}^{\mathrm{y}} ; m \in m^{\mathrm{y}}$, а также $s_{v}^{\mathrm{M}} \cap s_{w}^{\mathrm{M}}=\varnothing, v \neq w: \forall v, \forall w \in m^{\mathrm{M}}$.

Выражение (19) отвечает такому шифру содержательного модуля:

\begin{tabular}{|c|c|c|c|c|}
\hline \multirow[t]{5}{*}{ KXX-XX } & \multirow[t]{5}{*}{$\mathrm{XX}$} & \multirow[t]{5}{*}{$\mathrm{X}$} & \multirow[t]{5}{*}{$\mathrm{XX}$} & $\begin{array}{ll}\mathrm{XX} & \text { substantial number } \\
\text { of the module for this } \\
\text { competence }\end{array}$ \\
\hline & & & & $\begin{array}{l}\text { number skills of this } \\
\text { competence }\end{array}$ \\
\hline & & & & level of development of skill \\
\hline & & & & view of skill \\
\hline & & & & cipher of competence \\
\hline
\end{tabular}

$\mid$\begin{tabular}{l|l|} 
KXX-XX $|\mathrm{XX}| \mathrm{XX} \mid \begin{array}{l}\mathrm{XX} \text { номер содержательного } \\
\text { модуля, сквозной для } \\
\text { данной компетенции }\end{array}$ \\
$\begin{array}{l}\text { номер умения, сквозной для } \\
\text { данной компетенции }\end{array}$ \\
$\begin{array}{l}\text { уровень сформированности } \\
\text { умения }\end{array}$ \\
вид умения \\
шифр компетенции
\end{tabular}

Например, шифр 1.ПФ.Е.01.ПУ.Г.01.01 означает, что выпускник ВУЗа должны иметь 1-вую производственную функцию (проектную), которой отвечает профессиональньй вид типичной задачи деятельности (ПФ), их отображает эвристический класс типичной задачи деятельности (E), при этом выпускник, владея навыками разработки программных документов относительно управления системой обеспечения ИБ (01), при уровне сформированности умения (Р), то есть способности выполнять действие, опираясь на постоянный умственный контроль без помощи материальных носителей информации, имеет в условиях профессиональной деятельности в составе группы специалистов или единолично согласно действующим нормативно-правовым актам готовить проекть нормативных документов (стратегии, программы, концепиии, доктрины), исходя из всестороннего анализа и реальной оценки ситуации, ресурсного обеспечения и отбора адекватных механизмов внедрения управленческих решений (01) за счет знания характеристики современных социально-политических процессов в Украине; общегосударственной системы обеспечения ИБ (01).

Имея эти модели и алгоритмы можно реализовать информационно-образовательные системы, какие могли бы широко использоваться в образовательных учреждениях.

\section{4. Выводы}

1. Проведен анализ направлений модернизации образовательных проектов подготовки специалистов в области ИБ по образовательно-квалификационному уровню бакалавр, который позволил определить наиболее рациональные пути построения качественного образовательного процесса. Проанализирована структура и определены составляющие построения ОПП как одного из основных элементов нормативных документов ОСВОУ.

2. Разработана математическая модель требований к ОСВОУ в соответствии с образовательным проектом подготовки специалистов в области ИБ. Модель позволяет эффективно планировать учебный процесс, определять основные компоненты ОКХ и ОПП, состоящие из дисциплин и соответствующих компетенций, взаимодействующих между собой. Определены принципы формирования содержательных модулей и их блоков, позволяющие определять перечень востребованных компетенций. Данная модель дает возможность компонировать сбалансированные учебные циклы по нормативной части ОСВОУ. 


\section{Литература}

[1] Grytsyuk Yu.I., Rak T.Ye., Problemy zakhystu informatsiyi u strukturnykh pidrozdilakh MNS Ukraiyni, „Naukovyy visnyk NLTU Ukrayiny” Vol. 12.12, 2011, pp. 330-346.

[2] Dronova I.V., Modelirovanie prognozov pri upravlenii uchebnymi proektami, „Aviatsiynokocmichna tekhnika i tekhnologiya" Vol. 8.8, 2003, pp. 43-46.

[3] Zgurovs'kiy M.Z., Pankratova N.D., Osnovi sistemnogo analizu [Pidruchnik], K.: Vidavnicha grupa VNV, 2007, p. 544.

[4] Myerkusheva I.V., Teslya N.Yu., Struktura informatsiynikh vzayemodiy $v$ sistemakh rozpodilenogo upravlinnya proektami, „Upravlinnya rozvitkom skladnikh sistem", Vol. 6, 2011, pp. 47-50.

[5] Miroshnikov B.N., Bor'ba skiberprestupleniyami - odna iz sostavlyayushchikh informatsionnoy bezopasnosti Rossiyskoy Federatsii, [Elektronnyy resurs], http://www.crime-research.org/library/ Miros1.html

[6] Nozdrina L.V., Polotay O.I., Dosvid peredumov zaprovadzhennya innovatsiynikh osvitnikh proektiv $u$ VNZ, „Obshchie problemy, e-obrazovaniya $\mathrm{v}$ vysshikh uchebnykh zavedeniyakh", INCEL-08, 2008, pp. 1-6.
[7] Redchenko K.I., Audit strategichnikh upravlinskikh rishen', prognoziv ta proektiv, Lviv: Vid-vo LKA, 2001, p. 402.

[8] Stashevskiy Z.P., Grytsyuk Yu.I., Analiz dzherel zagroz informatsiynym sistemam na etapi initsiatsiyi proektu, „Visnik LDU BZhD” Vol. 8, 2013, pp. 67-74.

[9] Teslenko P.A., Garmonichnoe upravlenie proektami, „Garmonichnoe razvitie sistem - tretiy put' chelovechestva: Kollektivnaya monografiya po materialam trudov 1-go Mezhdunarodnogo kongressa", Odessa: Institut kreativnykh tekhnologiy, 2011, pp. 375-380.

[10] Teslenko P.A., Evolyutsionnaya teoriya i sinergetika $v$ upravlenii proektami, „Upravlinnya proektami ta rozvitok virobnitstva" Vol. 36 Issue 4, 2010, pp. 38-44.

[11] Pro zatverdzhennya Poryadku rozroblennya, zatverdzhennya ta vnesennya zmin do galuzevikh standartiv visshchoyi osvity, Postanova Kabinetu Ministriv Ukrayiny vid 05 veresnya 2012, No. 847.

[12] Galuzeviy standart vischoyi osviti Ukrayiny Osvitno-profesiyna programa bakalavra napryamu pidgotovki 6.170103 „Upravlinnya informatsiynoyu bezpekoyu”, 2010, p. 128.

Сташевский Захар Петрович - адъюнкт, Львовский государственный университет безопасности жизнедеятельности.

д-р техн. наук, проф Грыцюк Юрий Иванович - проф. кафедры программного обеспечения, Национальный университет «Львовская политехника». 